\title{
Accessory mitral valve tissue
}

INSERM

\section{Source}

INSERM. (1999). Orphanet: an online rare disease and orphan drug data base. Accessory mitral valve tissue. ORPHA:99061

Accessory mitral valve tissue is a congenital non-syndromic heart malformation defined as an accessory mitral valve leaflet or various accessory mitral valve structures. It may be asymptomatic or present at various ages with symptoms of left ventricular outflow tract obstruction, low cardiac output due to subaortic obstruction or congestive heart failure. In some cases, it may be a source of cardioembolism. The malformation may be isolated or associated with other congenital heart malformations. 